Research Paper

\title{
Fetal/Placental Weight Ratio in Term Japanese Pregnancy: Its Difference Among Gender, Parity, and Infant Growth
}

\author{
Yoshio Matsuda ${ }^{\bowtie}$, Masaki Ogawa ${ }^{2}$, Akihito Nakai ${ }^{3}$, Masako Hayashi ${ }^{3}$, Shoji Satoh ${ }^{4}$, Shigeki Matsubara ${ }^{5}$ \\ 1. Department of Obstetrics and Gynecology, Professor, International University of Health and Welfare Hospital, Professor, 537-3 Iguchi Nasushi- \\ obara, Tochigi 329-2763, Japan \\ 2. Department of Obstetrics and Gynecology, Tokyo Women's Medical University, Associate professor, Kawada-cho, 8-1, Shinjuku-ku, Tokyo \\ 162-8666, Japan \\ 3. Department of Obstetrics and Gynecology, Tama-Nagayama Hospital, Nippon Medical School, Professor, 1-7-1 Nagayama, Tama-City, Tokyo \\ 206-8512, Japan \\ 4. Maternal and Perinatal Care Center, Oita Prefectural Hospital, Director, Bunyo 476, Oita 870-8511, Japan \\ 5. Department of Obstetrics and Gynecology, Jichi Medical University, Professor, 3311-1 Shimotsuke, Tochigi 329-0498, Japan
}

$\triangle$ Corresponding author: Yoshio Matsuda, International University of Health and Welfare Hospital, 537-3 Iguchi Nasushiobara, Tochigi 329-2763, Japan. Tel.: +81-287-39-3060, Fax: +81-287-39-3001, E-mail: yoshiom2979@gmail.com

(C) 2015 Ivyspring International Publisher. Reproduction is permitted for personal, noncommercial use, provided that the article is in whole, unmodified, and properly cited. See http:/ /ivyspring.com/terms for terms and conditions.

Received: 2015.01.20; Accepted: 2015.02.27; Published: 2015.03.25

\begin{abstract}
Purpose: The "inappropriately heavy placenta" has been considered to be associated with various pregnancy disorders; however, data is scarce what factors affect it. To determine whether the following three affect it; (1) infant gender and mother's parity, (2) growth restriction, and (3) preeclampsia.

Methods: We employed fetal/placental weight ratio (F/P). Subjects consisted of 53,650 infants and their placentas from women who vaginally delivered singleton live term infants. First, we examined whether F/P differs among the infant's gender or mother's parity. We classified the population into 4 categories according to gender and parity: male, nulliparous $(n=7,431)$, male, multiparous $(n=7,859)$, female, nulliparous $(n=7,559)$, female, multiparous $(n=7,800)$, and, compared F/P among the four groups. Next, we determined whether F/P differs in "small" or "large" for gestational age (SGA or LGA) infants, compared with appropriate for gestational age infants. Last, we determined whether preeclampsia (representative disorder of SGA) affects F/P.

Results: (1) F/P significantly differed according to infant gender and parity: female and nulliparity had significantly smaller F/P. F/P was significantly smaller in (2) SGA infants, and (3) infants from preeclamptic mothers.

Conclusion: We for the first time showed that in Japanese term vaginally-delivered singleton population, the following three had significantly smaller F/P than controls thus had "inappropriately heavy placenta": (1) female gender and nulliparity, (2) SGA infants, and (3) infants from preeclamptic mothers. We recommend that these factors should be taken into account in evaluating placental weight. These data may also be useful for further clarifying the fetal-placental pathophysiology in these conditions.
\end{abstract}

Key words: fetal/placental weight (F/P) ratio; placental weight; preeclampsia; small for gestational age

\section{Introduction}

The placental weight $(\mathrm{PW})$ is closely associated with the birth weight [1], and their ratio $(\mathrm{F} / \mathrm{P})$, which is often used as an index of placental nutrient efficiency, has been discussed in relation to adverse per- inatal outcomes, such as perinatal death, non-reassuring fetal status and low Apgar scores [2,3]. Generally speaking, unduly heavy placenta, i.e., the placenta heavier than expected from the infant's 
weight, has been reported to be associated with adverse pregnancy outcome. For example, oversized placenta [4] or high F/P [5] increased the risk of poor pregnancy outcome [4]. In complicated pregnancies associated with a low birth weight, the placenta was relatively heavy compared with the fetal weight [6]. Such unduly heavy placenta, here referred to as "inappropriately heavy placenta", not only badly affected the current pregnancy outcome but also the future development of various disorders: small infants with large placentas showed a higher incidence of developing hypertension later in adult life [7].

The F/P may differ depending on various factors, such as ethnicity, gestational week, or mode of delivery. Thus, we must take these factors into account to evaluate whether individual placenta is heavy or not. In short, we must have fundamental data of $\mathrm{F} / \mathrm{P}$, which may differ among pregnancies with various backgrounds. We here attempted to determine the following three: whether F/P differs depending on (1) infant gender and mother's parity, (2) the presence/absence of small for gestational age (SGA), and (3) presence/absence of preeclampsia.

\section{Materials and Methods}

The study protocol was reviewed and approved by the Ethics Committee of Nihon Medical University, Tama-Nagayama Hospital. This study was a retrospective cohort study using data from the Japan Society of Obstetrics and Gynecology (JSOG) Database: Database consisted of mainly data from secondary or tertiary obstetric hospitals.

Detailed descriptions of the database have been published previously [8, 9]. In brief, a self-administered questionnaire, interview and medical records were used to collect information on the parity, maternal age at delivery, maternal height, body mass index before pregnancy, smoking habit, alcohol intake during pregnancy, medical history, history of treatment for infertility, major obstetric complications during pregnancy, weight gain during pregnancy, mode of delivery, infant sex, gestational length (weeks), induction of labor and mode of delivery. Attendants routinely performed data entry at the time of delivery. The data conformed to uniform coding specifications and diagnostic criteria for complications and were subject to rigorous quality checking. The JSOG provided the dataset for the study, where the quality control for the database was assessed. Thereafter, the data were edited and reviewed. The gestational age was determined based on the menstrual history, the prenatal examination, and ultrasound findings during early pregnancy (gestational sac diameter, crown rump length and biparietal diameter). The diagnosis of complicated pregnancies, such as those with preeclampsia was recorded in the database in a check-box format ('yes' or 'no'). Patients were diagnosed to have preeclampsia if they had systolic blood pressure $>140 \mathrm{mmHg}$ or diastolic pressure $>90 \mathrm{mmHg}$ with proteinuria occurring after the 20th week of gestation but resolving by the $12^{\text {th }}$ week postpartum. The pregnant women were stratified to have severe preeclampsia when they had hypertension (systolic blood pressure $>160 \mathrm{mmHg}$ or diastolic blood pressure $>110 \mathrm{mmHg}$ ) and proteinuria defined as either $>2 \mathrm{~g} / 24 \mathrm{~h}$ urine collection or $>3+$ on a dipstick, on at least two separate occasions without urinary tract infection.

The untrimmed placentas were weighted by a midwife shortly after delivery, with the membranes and umbilical cord attached. The birth weight of the infant was measured in grams. F/P was calculated by dividing birth weight by $\mathrm{PW}$ in grams.

The exclusion criteria included all of the following; gestational age at delivery less than 24 weeks and over 42 weeks, clinical obstetric complications involving the placenta, such as placental abruption and placenta previa, stillbirth, birth weight less than $250 \mathrm{~g}$ and deliveries with missing data for parity, gestational age, birth weight, PW or the infant's gender. As a result, a complete case analysis was possible, because we were careful to only include cases with complete medical records. Study population consisted of 53,650 placentas from women who vaginally delivered a singleton live infant between 37 and 41 weeks of gestation [10]. The evaluation of the birth weight was determined using the 'New Japanese neonatal anthropometric charts' [10], then was classified as follows: small for gestational age (SGA, less than 10th percentile, $\mathrm{n}=4,670$ ), appropriate for gestational age (AGA, 10th 90th percentile, $n=44,424$ ) and large for gestational age (LGA, over 90th percentile, $n=4,556$ ). From the AGA infants, controls were selected and defined as follows: no history of smoking or alcohol consumption, no history of treatment for infertility (including ovulation induction, artificial insemination from husband (AIH) or in vitro fertilization-embryo transfer (IVF-ET)), no medical complications, nor pregnancy complications. Four sets of groups were constructed according to the infants' gender and the mothers' parity (nulliparous or multiparous), with each control as follows: Group A: male, nulliparous $(n=7,431)$, Group B: male, multiparous $(n=7,859)$, Group C: female, nulliparous $(n=7,559)$, and Group D: female, multiparous $(n=7,800)$. Then we labeled them (AGA control) as control-A, control-B, control-C, and control-D.

First, we examined whether the $\mathrm{F} / \mathrm{P}$ differs among the infant gender or mother's parity. Next, we determined whether the $\mathrm{F} / \mathrm{P}$ differs in SGA or LGA 
infants, compared to AGA control infants. Last, we determined whether preeclampsia (representative disorder of SGA) affects the F/P.

The results were expressed as the means \pm standard deviation (SD) or statistical difference $(95 \%$ confidence interval, CI). The statistical analyses were performed using the SAS 9.1 software program (SAS Institute, Cary, NC). An analysis of variance for continuous variables, confirmed by Dunnet's method, and the Kruscal-Wallis test (the chi-square test) for categorical variables, confirmed by Tukey's method, were used for the statistical analyses, and analysis of covariance (ANCOVA) was also used for considering the effect of maternal age. Significant differences were considered to be present for values $<0.05$.

\section{Results}

\section{Comparison of the birth weight, PW and F/P ratios among the four sub-groups}

Table 1 shows the number of deliveries, the birth weight, PW and F/P among the four groups. These variables of SGA and LGA infants and infants born from severe preeclamptic mothers $(n=723)$ were also shown in Table 1. Table 2 shows the statistical comparisons of the $\mathrm{F} / \mathrm{P}$ among the four AGA control groups. In all categorized-groups (A to $\mathrm{D}), \mathrm{F} / \mathrm{P}$ significantly differed both between males vs. females and nulli- vs. multiparity. The F/P was smaller in female than male infants irrespective of nulli-or multiparity. The $\mathrm{F} / \mathrm{P}$ was smaller in infants/placentas from nulliparous than multiparous women.

By performing ANCOVA, it became clear that these differences were not affected by maternal age (data not shown).

\section{Comparison of the F/P: the SGA and the LGA vs. AGA control among the four sub-groups}

Table 3 shows the statistical comparisons of the $\mathrm{F} / \mathrm{P}$ between the SGA or LGA and AGA control among the four sub-groups. In all categorized-groups (A to D), F/P was significantly smaller in SGA than AGA controls, whereas $\mathrm{F} / \mathrm{P}$ did not significantly differ between LGA and AGA controls.

\section{Comparison of the F/P in infants born from severe preeclamptic mothers versus AGA control among the four sub-groups}

Table 3 also shows the statistical comparisons of the $\mathrm{F} / \mathrm{P}$ ratio between the infants born from severe preeclamptic mothers and AGA controls among the four sub-groups. In all categorized groups (A to D), $\mathrm{F} / \mathrm{P}$ was significantly smaller in the infants born from severe preeclamptic mothers than in AGA controls.
Table 1. Number of deliveries, birth weight, placental weight and $\mathrm{F} / \mathrm{P}$ among four sub-groups

\begin{tabular}{|c|c|c|c|c|}
\hline Sub-groups & $\begin{array}{l}\text { No. of } \\
\text { deliveries }\end{array}$ & $\begin{array}{l}\text { Birth } \\
\text { Weight } \\
\text { (g) }\end{array}$ & $\begin{array}{l}\text { Placental } \\
\text { Weight (g) }\end{array}$ & $\mathbf{F} / \mathbf{P}$ \\
\hline \multicolumn{5}{|l|}{ A. Male, nulliparous } \\
\hline (AGA, control-A) & 7,431 & $3,039(266)$ & $578.8(92.6)$ & $5.35(0.78)$ \\
\hline (SGA) & 1,209 & $2,370(285)$ & $461.9(92.6)$ & $5.29(0.97)$ \\
\hline (LGA) & 1,154 & $3,674(256)$ & $697.3(102.8)$ & $5.37(0.79)$ \\
\hline$(\mathrm{s}-\mathrm{PE})$ & 245 & $2,733(571)$ & $547.2(128.2)$ & $5.08(0.82)$ \\
\hline \multicolumn{5}{|c|}{ B. Male, multiparous } \\
\hline (AGA, control-B) & 7,859 & $3,142(279)$ & $592.7(96.6)$ & $5.41(0.79)$ \\
\hline (SGA) & 1,105 & $2,459(280)$ & $473.8(94.0)$ & $5.34(0.96)$ \\
\hline (LGA) & 1,139 & $3,775(261)$ & $715.1(106.4)$ & $5.38(0.82)$ \\
\hline (s-PE) & 117 & $2,810(610)$ & $558.3(139.4)$ & $5.14(0.87)$ \\
\hline \multicolumn{5}{|l|}{$\begin{array}{l}\text { C. Female, nullipa- } \\
\text { rous }\end{array}$} \\
\hline (AGA, control-C) & 7,559 & $2,964(258)$ & $570.8(91.4)$ & $5.30(0.78)$ \\
\hline$(\mathrm{SGA})$ & 1,228 & $2,315(275)$ & $462.6(90.7)$ & $5.14(0.90)$ \\
\hline (LGA) & 1,139 & $3,581(249)$ & $688.8(103.8)$ & $5.30(0.79)$ \\
\hline$(\mathrm{s}-\mathrm{PE})$ & 248 & $2,682(495)$ & $541.7(126.8)$ & $5.08(0.89)$ \\
\hline \multicolumn{5}{|l|}{$\begin{array}{l}\text { D. Female, multipa- } \\
\text { rous }\end{array}$} \\
\hline (AGA, control-D) & 7,800 & $3,042(271)$ & $580.0(95.1)$ & $5.35(0.79)$ \\
\hline$(\mathrm{SGA})$ & 1,128 & $2,378(286)$ & $470.9(92.9)$ & $5.19(0.93)$ \\
\hline (LGA) & 1,124 & $3,662(266)$ & 709.1 (106.2) & $5.27(0.80)$ \\
\hline (s-PE) & 113 & $2,764(617)$ & $573.7(147.8)$ & $4.93(0.82)$ \\
\hline \multicolumn{5}{|c|}{$\begin{array}{l}\text { LGA: large for gestational age, s-PE: severe preecla } \\
\text { F/P: birth weight/placental weight ratio }\end{array}$} \\
\hline
\end{tabular}

Table 2. Statistical comparison of F/P among four sub-groups in AGA control

\begin{tabular}{llll}
\hline Sub-groups & $\begin{array}{l}\text { B. Male, mul- } \\
\text { tiparaous }\end{array}$ & $\begin{array}{l}\text { C. Female, nullip- } \\
\text { arous }\end{array}$ & $\begin{array}{l}\text { D. Female, } \\
\text { multiparous }\end{array}$ \\
\hline A. Male, nulliparous & $-0.06^{*}$ & $0.06^{*}$ & $0 \#$ \\
& $(-0.09--0.02)$ & $(0.02-0.09)$ & $(-0.03-0.03)$ \\
B. Male, multiparous & $0.11^{*}$ & $0.06^{*}$ \\
& $(0.08-0.14)$ & $(0.02-0.09)$ \\
C. Female, nulliparous & & $-0.06^{*}$ \\
& & $(-0.08--0.02)$ \\
\hline AGA: appropriate for gestational age, & \\
F/P: birth weight/placental weight ratio & \\
Numerals indicates statistical difference $(95 \% \mathrm{CI})$ & \\
*: p<0.0001, \#: NS (not significant) &
\end{tabular}

Table 3. Statistical comparison of F/P among four sub-groups

\begin{tabular}{llll}
\hline Sub-groups & & & \\
\hline & $\begin{array}{l}\text { SGA vs. } \\
\text { AGA control }\end{array}$ & $\begin{array}{c}\text { LGA vs. } \\
\text { AGA control }\end{array}$ & $\begin{array}{c}\text { s-PE vs. } \\
\text { AGA control }\end{array}$ \\
\hline A. Male, nulliparous & $-0.06^{*}$ & $0.02^{\#}$ & $-0.28^{*}$ \\
& $(-0.02--0.11)$ & $(-0.03-0.06)$ & $(-0.17--0.38)$ \\
B. Male, multiparous & $-0.06^{*}$ & $-0.02^{\#}$ & $-0.27^{*}$ \\
& $(-0.02--0.12)$ & $(-0.07-0.03)$ & $(-0.13--0.42)$ \\
C. Female, nulliparous & $-0.16^{*}$ & $0.01^{\#}$ & $-0.22^{*}$ \\
& $(-0.11--0.20)$ & $(-0.04-0.05)$ & $(-0.12--0.32)$ \\
D. Female, multiparous & $-0.16^{*}$ & $-0.08^{\#}$ & $-0.42^{*}$ \\
& $(-0.11--0.21)$ & $(-0.03-0.13)$ & $(-0.28--0.57)$ \\
\hline
\end{tabular}

F/P: birth weight/placental weight ratio

AGA: appropriate for gestational age,

SGA: small for gestational age,

LGA: large for gestational age,

s-PE: severe preeclampsia

Numerals indicates statistical difference $(95 \% \mathrm{CI})$

${ }^{*}$ : p $<0.0001$, \#: NS (not significant) 


\section{Discussion}

We for the first time showed that in Japanese term vaginally-delivered singleton population, the following three had significantly smaller $F / P$ than controls: (1) female gender and nulliparity, (2) SGA infants, and (3) infants from preeclamptic mothers.

We believe that F/P should be established in an individual population and should be updated. The secular trends in neonatal anthropometric measurements at birth are associated with changes not only in antenatal management and maternal age and size, but also in socioeconomic or environmental conditions. Neonatal growth charts should be updated to reflect these changes [11]. This may also hold true to F/P. The major previous reports on $\mathrm{F} / \mathrm{P}$ were from 1970's [12], 1980's [4] or 1990's [13, 14]. In addition, these reports have the problem of ethnicities (multiracialities), and, thus, report on F/P based on single ethnicity is waited. Fortunately, more than $95 \%$ of the present study population consisted of single ethnicity (Japanese), and, thus, present data overcame the problem caused by heterogeneous ethnicities. Previous report showed F/P of Japanese population, but it was derived from a small sample size $(n=3,434)$ at a single institute [15]. We believe that the present data, at least partly, provided fundamental data of $\mathrm{F} / \mathrm{P}$ of single ethnicity based on a large sample size.

We here confined the study population to vaginal delivery. This is because pregnancy ending in cesarean section (CS) may consist of different population from that of vaginal delivery. Some data support this view. Skjaerven et al. [16] noted a clear difference in the birth weight for most of the preterm infants, which was possibly due to high incidence of CS in this population, and, thus, they, subsequently excluding the subjects delivered by CS, provided a new standard for SGA infants. Itabashi et al. [10] created new Japanese neonatal anthropometric charts, excluding cases delivered by CS, which represents an unrestricted growth pattern mimicking fetal growth. All these indicate that F/P may also be strongly influenced by the mode of delivery. It is highly expected that multifetal pregnancy and preterm delivery may also strongly affect $F / P$. Thus, we here confined the study population to term, singleton, vaginally delivered infants. We are aware that this is the first step to establish F/P in Japanese population: F/P should be determined not only to the present population but also to those of multifetal pregnancy, from abdominal deliveries, and preterm deliveries.

In the present study, the $\mathrm{F} / \mathrm{P}$ was significantly different depending on both parity and gender. The effects of gender and parity on the placental weight and $F / P$ have not been fully investigated. Wallace et al. [3] have recently reported that both gender and parity influence the placental weight percentiles: parity did not affect $\mathrm{F} / \mathrm{P}$ but gender affected it. The gender difference may reflect different metabolic programming between male vs. female [17], although its precise mechanism is unclear.

The F/P was smaller in the SGA than in the AGA control. The reason for this is unclear. Growth restricted infants demand more nutrition, which may cause overgrowth of the placenta. Or, the placenta of SGA infants is not well functioning and thus it becomes voluminous (overgrowth), leading to heavy placenta. The third mechanism associated with the enlargement of the placenta is caused by fetal hypoxemia either due to a reduced maternal blood oxygen content or due to ischemia and infarction of the placenta [18]; however, it is practically important to be aware that SGA infants have "inappropriately heavy placenta ". Different from SGA, F/P did not differ between LGA vs. AGA control. We do not know the reason for this phenomenon. The placenta may play smaller roles in LGA than in SGA at least from the viewpoint of $\mathrm{F} / \mathrm{P}$.

We have chosen preeclampsia as a representative and symbolic disorder causing SGA. In preeclamptic cases, $\mathrm{F} / \mathrm{P}$ was smaller than that of AGA control. Salafia et al. showed that inappropriately heavy placenta might be an expression of a relatively inefficient placenta with reduced ability to maintain fetal growth [19]. More plainly, the preeclamptic placenta, being functionally less effective to maintain the fetal growth compared with non-preeclamptic placenta, becomes large; thus, showing one aspect of a compensatory mechanism of the placenta.

This study has some potential limitations. First, the cord clamping practice and weighing the untrimmed placenta at birth could have affected the F/P. However, it has been reported that the correlation remained high between untrimmed placenta and placenta with the umbilical cord cut and membranes removed [20]. Second, although the study number was large, the present data did not cover all deliveries in Japan, different from the report from Norway [17].

\section{Conclusions}

We demonstrated that (1) female gender and nulliparity, (2) SGA infants, and (3) infants from preeclamptic mothers had inappropriately heavy placenta. We recommend that these factors should be taken into account in evaluating placental weight in an individual manner. These data may also be useful for further clarifying the fetal-placental pathophysiology in fetal growth restriction and its related condition. 


\section{Abbreviations}

PW: placental weight; F/P: birth weight/placental weight; JSOG: Japan Society of Obstetrics and Gynecology; SGA: small for gestational age; AGA: appropriate for gestational age; LGA: large for gestational age; AIH: artificial insemination from husband; IVF-ET: in vitro fertilization-embryo transfer; SD: standard deviation; CS: cesarean section; CI: confidence interval.

\section{Acknowledgements} help.

We thank Mr. Norio Sugimoto for his statistical

\section{Competing Interest}

The authors have declared that no competing interest exists.

\section{References}

1. Salafia CM, Zhang J, Charles AK, et al. Placental characteristics and birth weight. Paediatr Perinat Epidemiol 2008;22:229-239.

2. Molteni RA. Placental growth and fetal/placental weight $(F / P)$ ratios throughout gestation-their relationship to patterns of fetal growth. Semin Perinatol 1984;8:94-100

3. Wallace JM, Bhattacharya S, Horgan GW. Gestational age, gender and parity specific centile charts for placental weight for singleton deliveries in Aberdeen, UK. Placenta 2013;30: 269-274.

4. Naeye RL. Do placental weights have clinical significance? Hum Pathol 1987;18:387-391.

5. Bonds DR, Gabbe SG, Kumar S, Taylor T. Fetal weight/placental weight ratio and perinatal outcome. Am J Obstet Gynecol 1984;149:195-200.

6. Eskild A, Romundstad PR, Vatten LJ. Placental weight and birth weight: does the association differ between pregnancies with and without preeclampsia? Am J Obster Gynecol 2009;201:595-600

7. Barker DJ, Bull AR, Osmond C, Simmonds SJ. Fetal and placental size and risk of hypertension in adult life. BMJ 1990;301:259-262.

8. Matsuda M, Hayashi K, Shiozaki A, et al. Comparison of risk factors for placental abruption and placenta previa: case-cohort study. J Obstet Gynaecol Res. 2011;37:538-546.

9. Hayashi M, Nakai A, Satoh S, Matsuda Y. Adverse obstetric and perinatal outcomes of singleton pregnancies may be related to maternal factors associated with infertility rather than the type of assisted reproductive technology procedure used. Fertil Steril 2012;98:922-928.

10. Itabashi K, Miura F, Uehara R, Nakamura Y. New Japanese neonatal anthropometric charts for gestational age at birth. Pediatr Int 2014;56:702-708.

11. Bertino E, Milani S, Fabris CC, De Curtis M. Neonatal anthropometric charts: What they are, what they are not. Arch Dis Child Fetal Neonal Ed 2007;92:F7-10

12. Molteni RA, Stys SJ, Battaglia FC. Relationship of fetal and placental weight in human beings: Fetal/placental weight ratios at various gestational ages and birth weight distributions. J Reprod Med 1978;21: 327-334.

13. Godfrey KM, Redman CW, Barker DJ, Osmond C. The effect of maternal anemia and iron-deficiency on the ratio of fetal weight to placental weight. Br J Obstet Gynaecol 1991;98: 886-891.

14. Lao TT, Wong W. The neonatal implications of a high placental ratio in small-for gestational age infants. Placenta 1999;20:723-726.

15. Hasegawa J, Arakawa K, Nakamura M, et al. Analysis of placental weight centile is useful to estimate cause of fetal growth restriction. J Obstet Gynecol Res 2011;37:1658-1665.

16. Skjaerven R, Gjessing HK, Bakketeig LS. Birthweight by gestational age in Norway. Acta Obstet Gynecol Scand 2000;79:440-449.

17. Thompson JM, Irgens LM, Skjaerven R, Rasmussen S. Placenta weight percentile curves for singleton deliveries. BJOG 2007;114:715-720.

18. Luque-Fernandez MA, Ananth CV, Jaddoe VW, et al. Is the fetoplacental ratio a differential marker of fetal growth restriction in small for gestational age infants? Eur J Epidemiol DOI: 10.1007/s10654-015-9993-9

19. Salafia CM, Charles AK, Maas EM. Placenta and fetal growth restriction. Clin Obstet Gynecol 2006;49:236-256.

20. Leary SD, Godfrey KM, Greenaway LJ, Davill VA, Fall CH. Contribution of the umbilical cord and membranes to untrimmed placental weight. Placenta 2003;24:276-8. 